TRANSACTIONS OF THE

AMERICAN MATHEMATICAL SOCIETY

Volume 353, Number 1, Pages 269-289

S 0002-9947(00)02698-2

Article electronically published on September 15, 2000

\title{
SHARP SOBOLEV INEQUALITIES WITH LOWER ORDER REMAINDER TERMS
}

\author{
OLIVIER DRUET, EMMANUEL HEBEY, AND MICHEL VAUGON
}

\begin{abstract}
Given a smooth compact Riemannian $n$-manifold $(M, g)$, this paper deals with the sharp Sobolev inequality corresponding to the embedding of $H_{1}^{2}(M)$ in $L^{2 n /(n-2)}(M)$ where the $L^{2}$ remainder term is replaced by a lower order term.
\end{abstract}

Let $(M, g)$ be a smooth compact Riemannian $n$-dimensional manifold, $n \geq 3$. Let $H_{1}^{2}(M)$ be the standard Sobolev space, defined as the completion of $C^{\infty}(M)$ with respect to

$$
\|u\|_{H_{1}^{2}}^{2}=\|\nabla u\|_{2}^{2}+\|u\|_{2}^{2}
$$

where $\|\cdot\|_{p}, p \geq 1$, stands for the $L^{p}$-norm. By the Sobolev embedding theorem, one has that $H_{1}^{2}(M) \subset L^{2^{\star}}(M)$, where $2^{\star}=2 n /(n-2)$ is critical. Given $\theta \in[1,2]$, there exist $A, B \in \mathbb{R}$ such that, for any $u \in H_{1}^{2}(M)$,

$$
\|u\|_{2^{\star}}^{2} \leq A\|\nabla u\|_{2}^{2}+B\|u\|_{\theta}^{2}
$$

This easily follows from the Sobolev-Poincaré inequality. Now define $K_{n}$ as the best constant $K$ for the standard $H_{1}^{2}$-Sobolev inequality in $\mathbb{R}^{n}$ :

$$
\|u\|_{2^{\star}} \leq K\|\nabla u\|_{2}
$$

As is well known, see [1] and [24],

$$
K_{n}=\sqrt{\frac{4}{n(n-2) \omega_{n}^{2 / n}}}
$$

where $\omega_{n}$ stands for the volume of the unit sphere $S^{n}$ of $\mathbb{R}^{n+1}$. Mimicking what was done in [15], proposition 4.5, one easily checks that any constant $A$ in $\left(S_{\theta}\right)$ has to be greater than or equal to $K_{n}^{2}$. Conversely, one may prove that for any $\varepsilon>0$, there exists $B_{\varepsilon}$ such that for any $u \in H_{1}^{2}(M)$,

$$
\|u\|_{2^{\star}}^{2} \leq\left(K_{n}^{2}+\varepsilon\right)\|\nabla u\|_{2}^{2}+B_{\varepsilon}\|u\|_{\theta}^{2}
$$

This follows from standard arguments, by locally comparing the Riemannian metric with the Euclidean metric, and then by gluing together the local inequalities to get the global one.

These few remarks lead to the consideration of the following sharp inequality: for any $u \in H_{1}^{2}(M)$,

$$
\left(S_{\text {opt }}^{\theta}\right) \quad\|u\|_{2^{\star}}^{2} \leq K_{n}^{2}\|\nabla u\|_{2}^{2}+B\|u\|_{\theta}^{2}
$$

Received by the editors June 15, 1999.

2000 Mathematics Subject Classification. Primary 58E35. 
where $B \in \mathbb{R}$. In what follows, we say that $\left(S_{\text {opt }}^{\theta}\right)$ is valid on $(M, g)$ if there exists $B \in \mathbb{R}$ such that $\left(S_{o p t}^{\theta}\right)$ holds for all $u \in H_{1}^{2}(M)$. As one can easily check, if $\left(S_{o p t}^{\theta}\right)$ is valid for some $\theta_{0} \in[1,2]$, then it is also valid for all $\theta \in\left[\theta_{0}, 2\right]$. For $\theta=1$, one recovers a sharp inequality of Sobolev-Poincaré type.

Given $\theta \in[1,2]$, and $(M, g)$ a smooth compact Riemannian $n$-manifold, $n \geq 3$, this article deals with the question of how we can know whether $\left(S_{o p t}^{\theta}\right)$ is valid on $(M, g)$ or not. Among others, such a question was considered in Aubin and Li 3]. Since the work of Druet [10, we know that a key point when studying sharp Sobolev inequalities is to prove that such inequalities are localisable. They may then be affected by the geometry. In other words, sometimes they are valid and sometimes not, depending on the manifold. This will be referred to as the effect of geometry. In addition to the original [10, see also [1] and [12]. Another effect that may occur when studying elliptic type problems, first pointed out by Brezis and Nirenberg in their celebrated paper [6], is the so-called low dimensions effect: a situation may change when passing from low dimensions to high dimensions. A particularly interesting feature when studying the scale $\left(S_{o p t}^{\theta}\right)$ of sharp inequalities, $\theta \in[1,2]$, is that it mixes both types of phenomena. For $\theta=2$, we know by Hebey and Vaugon [19] that $\left(S_{\text {opt }}^{2}\right)$ is always valid, without any assumptions on the manifold. For $\theta<2$, it appears that $\left(S_{o p t}^{\theta}\right)$ is always localisable, with the property that it is affected by the geometry when $n \geq 4$, but not when $n=3$.

Concerning the terminology, a Cartan-Hadamard $n$-dimensional manifold is a complete simply-connected $n$-dimensional Riemannian manifold of nonpositive sectional curvature. We then refer to the $n$-dimensional Cartan-Hadamard conjecture as follows: given a Cartan-Hadamard $n$-dimensional manifold $(\tilde{M}, \tilde{g})$ and a smooth bounded domain $\Omega$ in $\tilde{M}$, we ask for the inequality

$$
\frac{\operatorname{Area}_{\tilde{g}}(\partial \Omega)}{\operatorname{Vol}_{\tilde{g}}(\Omega)^{(n-1) / n}} \geq n\left(\frac{\omega_{n-1}}{n}\right)^{\frac{1}{n}}
$$

that is the value one gets for the above ratio in the Euclidean $n$-dimensional space when $\Omega$ is a ball. Such a conjecture has been proved to be true for $n=2$ by Weil [26], for $n=3$ by Kleiner [22], and for $n=4$ by Croke [8]. Our first result is the following. We let $S_{g}$ and $K_{g}$ be the scalar and sectional curvatures of $g$.

Theorem 1. Let $(M, g)$ be a smooth compact Riemannian $n$-manifold, $n \geq 3$. For $\theta=2,\left(S_{o p t}^{2}\right)$ is valid on $(M, g)$ without any assumption. On the contrary, for $\theta \in[1,2),\left(S_{\text {opt }}^{\theta}\right)$ is valid on $(M, g)$ if either $K_{g}$ is a nonpositive constant, or $K_{g}$ is nonpositive everywhere on $M$ and the $n$-dimensional Cartan-Hadamard conjecture is true, but $\left(S_{\text {opt }}^{\theta}\right)$ is false if $n \geq 4$ and $S_{g}$ is positive somewhere on $M$.

A main step in the proof of this theorem is to show that $\left(S_{o p t}^{\theta}\right)$ is localisable - in other words to prove that if for any $x \in M$, there exist an open neighborhood $\Omega_{x}$ of $x$, and $B_{x} \in \mathbb{R}$, such that for any $u \in C_{c}^{\infty}\left(\Omega_{x}\right)$,

$$
\|u\|_{2^{\star}}^{2} \leq K_{n}^{2}\|\nabla u\|_{2}^{2}+B_{x}\|u\|_{\theta}^{2}
$$

then $\left(S_{o p t}^{\theta}\right)$ is valid on $(M, g)$. For $\theta=2$, a partition of unity easily gives the localisation. For $\theta<2$, it is proved here by PDE type arguments.

Regarding Theorem 1, one sees that for $n \geq 4$, and $\theta<2,\left(S_{o p t}^{\theta}\right)$ is valid depending on the sign of the curvature. This is the illustration in our context of the first type of phenomenon mentioned above: the effect of geometry. For $n=3$, as we see in Theorem 2 below, $\left(S_{\text {opt }}^{\theta}\right)$ is valid for any $\theta$ on any manifold. One 
recovers there the second type of phenomenon mentioned above: the effect of low dimensions. Theorems 1 and 2 were announced in [13].

Theorem 2. For any smooth compact Riemannian 3-manifold $(M, g)$, and for any $\theta \in[1,2],\left(S_{\text {opt }}^{\theta}\right)$ is valid on $(M, g)$.

Now define $B_{0}(g)$ in $\left(S_{\text {opt }}^{2}\right)$ by

$$
B_{0}(g)=\inf \left\{B \in \mathbb{R} \text { for which }\left(S_{\text {opt }}^{2}\right) \text { is true }\right\}
$$

Consider then the optimal Sobolev inequality: for all $u \in H_{1}^{2}(M)$,

$$
\left(S_{O P T}^{2}\right) \quad\|u\|_{2^{\star}}^{2} \leq K_{n}^{2}\|\nabla u\|_{2}^{2}+B_{0}(g)\|u\|_{2}^{2}
$$

Such an inequality is always valid. By definition, we say that $u \in H_{1}^{2}(M), u \neq \equiv 0$, is an extremal function for $\left(S_{O P T}^{2}\right)$ if $u$ realizes the equality in $\left(S_{O P T}^{2}\right)$. The existence of extremal functions for $\left(S_{O P T}^{2}\right)$ was studied by Hebey [16, and more recently by Djadli and Druet [9]. It was proved in [16] that if $n \geq 4$, and $g$ is a conformal metric to the standard metric $h$ on the unit sphere $S^{n}$, then $\left(S_{O P T}^{2}\right)$ with respect to $g$ has extremal functions if and only if $g$ and $h$ are (up to a constant) isometric. In particular, there exist manifolds of positive scalar curvature for which $\left(S_{O P T}^{2}\right)$ does not have extremal functions. Among other results, it was proved in [9] that when $n \geq 4,\left(S_{O P T}^{2}\right)$ possesses extremal functions if either $S_{g}$ is nonpositive, or $S_{g}$ is constant. As a concluding remark, following an idea that was developed by Humbert [21], and quoting a theorem that was stated in the very recent announcement [18] by Hebey, Humbert and Vaugon, we wish to point out the influence that $\left(S_{o p t}^{1}\right)$ has on the existence of extremal functions for the optimal Sobolev inequality $\left(S_{O P T}^{2}\right)$. This is the subject of Theorem 3 below.

Theorem 3. Let $(M, g)$ be a smooth compact Riemannian $n$-manifold, $n \geq 5$. Suppose that $\left(S_{\text {opt }}^{1}\right)$ is true. Then there exist extremal functions for $\left(S_{O P T}^{2}\right)$. In particular, $\left(S_{O P T}^{2}\right)$ possesses extremal functions on flat tori and compact hyperbolic spaces, $n \geq 5$, or again on compact Riemannian $n$-manifolds of nonpositive sectional curvature, $n \geq 5$, if the $n$-dimensional Cartan-Hadamard conjecture is true.

The rest of the article is devoted to the proof of these three theorems.

\section{Proof of Theorem 1}

As already mentioned, a main step when proving Theorem 1 is to show that $\left(S_{o p t}^{\theta}\right)$ is localisable. This is done through PDE arguments. A preliminary technical lemma is needed.

Lemma 1. Let $(M, g)$ be a smooth compact Riemannian $n$-manifold, $n \geq 3$, and let $u \in H_{1}^{2}(M), u \geq 0$, be such that for any nonnegative $\varphi \in H_{1}^{2}(M)$,

$$
\int_{M}\langle\nabla u, \nabla \varphi\rangle d v_{g} \leq \int_{M} u^{2^{\star}-1} \varphi d v_{g}
$$

Then $u \in L^{\infty}(M)$. Moreover, for any $x$ in $M$, any $\Lambda>0$, any $p>0$, and any $q>2^{\star}$, there exists $\delta>0$ such that if $u$ is some nonnegative function of $H_{1}^{2}(M)$ satisfying (1.1) and

$$
\int_{B_{x}(2 \delta)} u^{q} d v_{g} \leq \Lambda
$$


then

$$
\sup _{y \in B_{x}(\delta)} u(y) \leq \tilde{C}\left(\int_{B_{x}(2 \delta)} u^{p} d v_{g}\right)^{1 / p}
$$

where $\tilde{C}>0$ does not depend on $u$.

We omit the proof of this lemma since it goes in a very standard way. Mimicking what was done in Trudinger 25] when studying the regularity for the Yamabe problem, one first gets that $u \in L^{k}(M)$ for some $k>2^{\star}$. The lemma then follows from the classical De Giorgi-Nash-Moser theory when studying inequations of the type

$$
\Delta_{g} u+c u \leq f
$$

where $\Delta_{g} u=-\operatorname{div}_{g}(\nabla u)$ stands for the Laplacian of $u$ with respect to $g$. We let $c=-u^{2^{\star}-2}$ and $f=0$ here.

Going on with Theorem 1, we prove now that $\left(S_{\text {opt }}^{\theta}\right)$ is localisable.

Lemma 2. Let $(M, g)$ be a smooth compact Riemannian $n$-manifold, $n \geq 3$, and let $\theta \in[1,2]$. Suppose that for any $x$ in $M$, there exist an open neighborhood $\Omega_{x}$ of $x$, and $B_{x} \in \mathbb{R}$, such that for any $u \in C_{c}^{\infty}\left(\Omega_{x}\right)$,

$$
\|u\|_{2^{\star}}^{2} \leq K_{n}^{2}\|\nabla u\|_{2}^{2}+B_{x}\|u\|_{\theta}^{2}
$$

Then $\left(S_{o p t}^{\theta}\right)$ is valid on $(M, g)$.

Proof. For any $\alpha>0$, let $I_{\alpha}$ be the functional defined on $H_{1}^{2}(M) \backslash\{0\}$ by

$$
I_{\alpha}(u)=\frac{\|\nabla u\|_{2}^{2}+\alpha\|u\|_{\theta}^{2}}{\|u\|_{2^{\star}}^{2}}
$$

Under the assumptions of the lemma, namely that $\left(S_{o p t}^{\theta}\right)$ is locally valid, the lemma reduces to the existence of some $\alpha_{0}$ such that

$$
\inf _{H_{1}^{2}(M) \backslash\{0\}} I_{\alpha_{0}}(u) \geq \frac{1}{K_{n}^{2}}
$$

We proceed by contradiction, and assume that for any $\alpha>0$,

$$
\inf _{H_{1}^{2}(M) \backslash\{0\}} I_{\alpha}(u)<\frac{1}{K_{n}^{2}}
$$

The proof then goes in several steps.

Step 1 . We claim that (1.2) implies the existence of a nonnegative minimizer for

$$
\mu_{\alpha}=\inf _{H_{1}^{2}(M) \backslash\{0\}} I_{\alpha}(u)
$$

If $\theta>1$, the proof of such a claim is quite standard. If $\theta=1$, one may proceed as follows. For $q<2^{\star}$, let $\theta_{q}>1$ be given with the property that $\theta_{q}$ goes to 1 as $q$ goes to $2^{\star}$. We fix $\alpha>0$ and for $q<2^{\star}$ we let

$$
\lambda_{q}=\inf _{H_{1}^{2}(M) \backslash\{0\}} \frac{\|\nabla u\|_{2}^{2}+\alpha\|u\|_{\theta_{q}}^{2}}{\|u\|_{q}^{2}}
$$

Since the embedding of $H_{1}^{2}(M)$ in $L^{q}(M)$ is compact, and since the above functional is homogeneous, there exists a nonnegative minimizer $u_{q}$ for $\lambda_{q}$ such that $\left\|u_{q}\right\|_{q}=1$. 
Clearly, $u_{q}$ is a weak solution of

$$
\Delta_{g} u_{q}+\alpha\left(\int_{M} u_{q}^{\theta_{q}} d v_{g}\right)^{\frac{2}{\theta_{q}}-1} u_{q}^{\theta_{q}-1}=\lambda_{q} u_{q}^{q-1}
$$

where $\Delta_{g}$ stands for the Laplacian with respect to $g$. As one can easily check, up to a subsequence we may assume that for some $\lambda_{\alpha} \leq \mu_{\alpha}$, the sequence $\left(\lambda_{q}\right)$ goes to $\lambda_{\alpha}$ as $q$ goes to $2^{\star}$. Noting that $\left(u_{q}\right)$ is bounded in $H_{1}^{2}(M)$, we see that there exists $u_{\alpha} \in H_{1}^{2}(M)$ such that, up to a subsequence, $\left(u_{q}\right)$ converges weakly to $u_{\alpha}$ in $H_{1}^{2}(M)$, strongly to $u_{\alpha}$ in $L^{2}(M)$, and almost everywhere. Moreover, one can assume that

$$
u_{q}^{q-1} \rightarrow u_{\alpha}^{2^{\star}-1} \text { in } L^{2^{\sharp}}(M)
$$

where $2^{\sharp}=2 n /(n+2)$ is the conjugate exponent of $2^{\star}$. By (1.2), and since for any $\varepsilon>0$ there exists $B_{\varepsilon}$ such that for any $u \in H_{1}^{2}(M)$,

$$
\|u\|_{2^{\star}}^{2} \leq\left(K_{n}^{2}+\varepsilon\right)\|\nabla u\|_{2}^{2}+B_{\varepsilon}\|u\|_{1}^{2}
$$

one has that $u_{\alpha} \not \equiv 0$. This is by now standard. Let $\varepsilon_{q}=\theta_{q}-1$. Clearly, $\left(u_{q}^{\varepsilon_{q}}\right)$ is bounded in $L^{p}(M)$ for any $p>1$. Concerning such an assertion, just note that for $q \gg 1$

$$
\left(\int_{M} u_{q}^{p \varepsilon_{q}} d v_{g}\right)^{1 / p} \leq\left(\int_{M} u_{q}^{2} d v_{g}\right)^{\varepsilon_{q} / 2} V_{g}^{\frac{1}{p}-\frac{\varepsilon_{q}}{2}}
$$

where $V_{g}$ stands for the volume of $M$ with respect to $g$. Since $L^{p}$-spaces are reflexive for $p>1$, there exists $\Sigma_{\alpha} \in \bigcap_{p>1} L^{p}(M)$ such that for any $p>1$, and up to a subsequence,

$$
u_{q}^{\varepsilon_{q}} \rightarrow \Sigma_{\alpha} \text { in } L^{p}(M)
$$

Passing to the limit as $q$ goes to $2^{\star}$ in (1.4), one gets that for any $p>1$,

$$
\left\|\Sigma_{\alpha}\right\|_{p} \leq V_{g}^{1 / p}
$$

As an easy consequence, $\Sigma_{\alpha} \in L^{\infty}(M)$ and $0 \leq \Sigma_{\alpha} \leq 1$. Another easy claim is that $\Sigma_{\alpha} \varphi=\varphi$ for any $\varphi \in H_{1}^{2}(M)$ having the property that $|\varphi| \leq C u_{\alpha}$ on $M$ for some constant $C>0$. By passing to the limit in (1.3), one gets that $u_{\alpha}$ is a weak solution of

$$
\Delta_{g} u_{\alpha}+\alpha\left(\int_{M} u_{\alpha} d v_{g}\right) \Sigma_{\alpha}=\lambda_{\alpha} u_{\alpha}^{2^{\star}-1}
$$

Clearly, $\left\|u_{\alpha}\right\|_{2^{\star}} \leq 1$. Mutiplying (1.5) by $u_{\alpha}$ and integrating over $M$ gives

$$
\frac{\left\|\nabla u_{\alpha}\right\|_{2}^{2}+\alpha\left\|u_{\alpha}\right\|_{2}^{2}}{\left\|u_{\alpha}\right\|_{2^{\star}}^{2}}=\lambda_{\alpha}\left\|u_{\alpha}\right\|_{2^{\star}}^{2^{\star}-2}
$$

As one can easily check, this implies that $\left\|u_{\alpha}\right\|_{2^{\star}}=1$ and $\lambda_{\alpha}=\mu_{\alpha}$. In particular, $u_{\alpha}$ is a minimizer for $\mu_{\alpha}$. The above claim is proved.

From now on, let $u_{\alpha} \in H_{1}^{2}(M), u_{\alpha} \geq 0, u_{\alpha} \not \equiv 0$, be the minimizer for $\mu_{\alpha}$ given by step 1 . As shown in the proof of step 1 , we can choose $u_{\alpha}$ such that

$$
\int_{M} u_{\alpha}^{2^{\star}} d v_{g}=1
$$

and for some $\Sigma_{\alpha} \in L^{\infty}(M)$, with the property that $0 \leq \Sigma_{\alpha} \leq 1, u_{\alpha}$ is a solution of

$$
\Delta_{g} u_{\alpha}+\alpha\left(\int_{M} u_{\alpha}^{\theta} d v_{g}\right)^{\frac{2}{\theta}-1} \Sigma_{\alpha}=\mu_{\alpha} u_{\alpha}^{2^{\star}-1}
$$


Moreover, $\Sigma_{\alpha}=u_{\alpha}^{\theta-1}$ if $\theta>1$, and if $\theta=1$, then $\Sigma_{\alpha} \varphi=\varphi$ for any $\varphi \in H_{1}^{2}(M)$ having the property that $|\varphi| \leq C u_{\alpha}$ on $M$ for some constant $C>0$. By lemma 1 , $u_{\alpha} \in L^{\infty}(M)$. Hence, $u_{\alpha} \in H_{2}^{p}(M)$ for any $p>1$, and $u_{\alpha}$ is actually in $C^{1, \lambda}$ for any $\lambda \in(0,1)$. As another remark, the sequence $\left(u_{\alpha}\right)$ is bounded in $H_{1}^{2}(M)$.

By definition (this is by now classical) we say that $x \in M$ is a concentration point for the sequence $\left(u_{\alpha}\right)$ if for any $\delta>0$,

$$
\limsup _{\alpha \rightarrow+\infty} \int_{B_{x}(\delta)} u_{\alpha}^{2^{\star}} d v_{g}>0
$$

Since $M$ is compact, the existence of at least one such point is easy to get. We prove the uniqueness of the concentration point in step 2 .

Step 2. We claim that, up to a subsequence, $\left(u_{\alpha}\right)$ has one and only one concentration point. Given $x \in M$ and $\delta>0, \delta$ small, let $\eta \in C_{c}^{\infty}\left(B_{x}(\delta)\right)$ be such that $0 \leq \eta \leq 1$ and $\eta=1$ in $B_{x}(\delta / 2)$. Multiplying $\left(E_{\alpha}\right)$ by $\eta^{2} u_{\alpha}^{k}, k \geq 1$ real, and integrating over $M$ lead to

$$
\begin{aligned}
& \int_{M} \eta^{2} u_{\alpha}^{k} \Delta_{g} u_{\alpha} d v_{g}+\alpha\left(\int_{M} u_{\alpha}^{\theta} d v_{g}\right)^{\frac{2}{\theta}-1} \int_{M} \eta^{2} u_{\alpha}^{k+\theta-1} d v_{g} \\
& \quad=\mu_{\alpha} \int_{M} \eta^{2} u_{\alpha}^{2^{\star}+k-1} d v_{g}
\end{aligned}
$$

As one can easily check,

$$
\begin{aligned}
& \int_{M} \eta^{2} u_{\alpha}^{k} \Delta_{g} u_{\alpha} d v_{g}=\frac{4 k}{(k+1)^{2}} \int_{M}\left|\nabla\left(\eta u_{\alpha}^{(k+1) / 2}\right)\right|^{2} d v_{g} \\
& -\frac{2(k-1)}{(k+1)^{2}} \int_{M} \eta\left(\Delta_{g} \eta\right) u_{\alpha}^{k+1} d v_{g}-\frac{2}{k+1} \int_{M}|\nabla \eta|^{2} u_{\alpha}^{k+1} d v_{g}
\end{aligned}
$$

while, by Hölder's inequality,

$$
\int_{M} \eta^{2} u_{\alpha}^{2^{\star}+k-1} d v_{g} \leq\left(\int_{M}\left(\eta u_{\alpha}^{(k+1) / 2}\right)^{2^{\star}} d v_{g}\right)^{2 / 2^{\star}}\left(\int_{B_{x}(\delta)} u_{\alpha}^{2^{\star}} d v_{g}\right)^{\left(2^{\star}-2\right) / 2^{\star}}
$$

According to Hebey and Vaugon [19], there exists $B>0$ such that for any $u \in$ $H_{1}^{2}(M)$

$$
\left(\int_{M}|u|^{2^{\star}} d v_{g}\right)^{2 / 2^{\star}} \leq K_{n}^{2} \int_{M}|\nabla u|^{2} d v_{g}+B \int_{M} u^{2} d v_{g}
$$

Coming back to (1.6), and since the second term in the left hand side of (1.6) is nonnegative, one gets that

$$
\begin{aligned}
& A_{\alpha}(k, \delta)\left(\int_{M}\left(\eta u_{\alpha}^{(k+1) / 2}\right)^{2^{\star}} d v_{g}\right)^{2 / 2^{\star}} \leq \frac{k-1}{2 k} K_{n}^{2} \int_{M} \eta\left(\Delta_{g} \eta\right) u_{\alpha}^{k+1} d v_{g} \\
& \quad+\frac{k+1}{2 k} K_{n}^{2} \int_{M}|\nabla \eta|^{2} u_{\alpha}^{k+1} d v_{g}+B \int_{M} \eta^{2} u_{\alpha}^{k+1} d v_{g}
\end{aligned}
$$

where

$$
A_{\alpha}(k, \delta)=1-\frac{(k+1)^{2}}{4 k} \mu_{\alpha} K_{n}^{2}\left(\int_{B_{x}(\delta)} u_{\alpha}^{2^{\star}} d v_{g}\right)^{\left(2^{\star}-2\right) / 2^{\star}}
$$

Suppose now that $x$ is a concentration point for $\left(u_{\alpha}\right)$. Given $\delta>0$, let

$$
\limsup _{\alpha \rightarrow+\infty} \int_{B_{x}(\delta)} u_{\alpha}^{2^{\star}} d v_{g}=\lambda_{\delta}
$$


Then $\lambda_{\delta}>0$ and $\lambda_{\delta} \leq 1$. Assume that $\lambda_{\delta}<1$ for some $\delta>0$. Together with (1.2), we may then choose $k>1$ sufficiently close to 1 such that

$$
1-\frac{(k+1)^{2}}{4 k} \mu_{\alpha} K_{n}^{2} \lambda_{\delta}^{\left(2^{\star}-2\right) / 2^{\star}}>0
$$

The right hand side of (1.7) being bounded for $k>1$ close to 1 , we get with (1.7) the existence of $K>0$ such that for $\alpha \gg 1$,

$$
\int_{M}\left(\eta u_{\alpha}^{(k+1) / 2}\right)^{2^{\star}} d v_{g} \leq K
$$

By Hölder's inequality, writing $2^{\star}=\left(2^{\star}-k-1\right)+(k+1)$,

$$
\begin{aligned}
\int_{B_{x}(\delta / 2)} u_{\alpha}^{2^{\star}} d v_{g} & \leq\left(\int_{M} u_{\alpha}^{2^{\star}-\frac{2^{\star}(k-1)}{2^{\star}-2}} d v_{g}\right)^{\left(2^{\star}-2\right) / 2^{\star}}\left(\int_{M}\left(\eta u_{\alpha}^{(k+1) / 2}\right)^{2^{\star}} d v_{g}\right)^{2 / 2^{\star}} \\
& \leq K^{2 / 2^{\star}}\left(\int_{M} u_{\alpha}^{2^{\star}-\frac{2^{\star}(k-1)}{2^{\star}-2}} d v_{g}\right)^{\left(2^{\star}-2\right) / 2^{\star}}
\end{aligned}
$$

Noting that for $k>1$ close to 1 ,

$$
1<2^{\star}-\frac{2^{\star}(k-1)}{2^{\star}-2}<2^{\star}
$$

one gets

$$
\lim _{\alpha \rightarrow+\infty} \int_{B_{x}(\delta / 2)} u_{\alpha}^{2^{\star}} d v_{g}=0
$$

Concerning such an assertion, just note that multiplying $\left(E_{\alpha}\right)$ by $u_{\alpha}$ and integrating over $M$ gives that the $L^{1}$-norm of $u_{\alpha}$ goes to 0 as $\alpha$ goes to $+\infty$, so that the $L^{q_{-}}$ norm of $u_{\alpha}$ also goes to 0 for any $1<q<2^{\star}$ (by Hölder's inequality and since the $L^{2^{\star}}$-norm of $u_{\alpha}$ is 1 ). Since (1.8) contradicts the definition of a concentration point, one actually has that $\lambda_{\delta}=1$ for any $\delta>0$. As one will easily check, up to the extraction of a subsequence, this implies that a concentration point must be unique. The above claim is proved.

We let $x_{0}$ be the concentration point for $\left(u_{\alpha}\right)$. Given $x \neq x_{0}$, one gets with (1.7) that for $\delta>0$ small, the $L^{\left(2^{\star}\right)^{2} / 2}$-norm of $u_{\alpha}$ in $B_{x}(\delta)$ is bounded. Together with lemma 1 , noting that $\left(2^{\star}\right)^{2} / 2>2^{\star}$, this implies that

$$
u_{\alpha} \rightarrow 0 \text { in } C_{l o c}^{0}\left(M \backslash\left\{x_{0}\right\}\right)
$$

as $\alpha$ goes to $+\infty$. Mimicking what was done in Druet [10, the final argument in the proof of lemma 2 goes in the following way.

Step 3. We claim that (1.2) is impossible for $\alpha$ sufficiently large. By assumption there exist $B \in \mathbb{R}$ and $\delta>0$ such that for any $u \in H_{0,1}^{2}\left(B_{\delta}\right)$,

$$
\left(\int_{M}|u|^{2^{\star}} d v_{g}\right)^{2 / 2^{\star}} \leq K_{n}^{2} \int_{M}|\nabla u|^{2} d v_{g}+B\left(\int_{M}|u|^{\theta} d v_{g}\right)^{2 / \theta}
$$

where $B_{\delta}=B_{x_{0}}(\delta)$ and $H_{0,1}^{2}\left(B_{\delta}\right)$ stands for the completion of $C_{c}^{\infty}\left(B_{\delta}\right)$ with respect to $\|\cdot\|_{H_{1}^{2}}$. We let $\eta \in C_{c}^{\infty}\left(B_{\delta}\right)$ be such that $0 \leq \eta \leq 1$ and $\eta=1$ in $B_{\delta^{\prime}}$ for some $\delta^{\prime} \in(0, \delta)$. If we set $\eta^{\prime}=1-\eta,(1.10)$ leads in particular to

$$
\left(\int_{B_{\delta^{\prime}}} u_{\alpha}^{2^{\star}} d v_{g}\right)^{2 / 2^{\star}} \leq K_{n}^{2} \int_{M}\left|\nabla\left(\left(1-\eta^{\prime}\right) u_{\alpha}\right)\right|^{2} d v_{g}+B\left(\int_{M} u_{\alpha}^{\theta} d v_{g}\right)^{2 / \theta}
$$


Clearly, there exists $C>0$, independent of $\alpha$, such that

$$
\begin{aligned}
\int_{M}\left|\nabla\left(\left(1-\eta^{\prime}\right) u_{\alpha}\right)\right|^{2} d v_{g} \leq & \int_{M}\left|\nabla u_{\alpha}\right|^{2} d v_{g}+C \int_{M \backslash B_{\delta^{\prime}}}\left|\nabla u_{\alpha}\right|^{2} d v_{g} \\
& +C \int_{M \backslash B_{\delta^{\prime}}} u_{\alpha}\left|\nabla u_{\alpha}\right| d v_{g}+C \int_{M \backslash B_{\delta^{\prime}}} u_{\alpha}^{2} d v_{g}
\end{aligned}
$$

Multiplying $\left(E_{\alpha}\right)$ by $u_{\alpha}$, and integrating over $M$, gives

$$
\int_{M}\left|\nabla u_{\alpha}\right|^{2} d v_{g}+\alpha\left(\int_{M} u_{\alpha}^{\theta} d v_{g}\right)^{2 / \theta}=\mu_{\alpha}
$$

Hence,

$$
\begin{aligned}
\left(\int_{B_{\delta^{\prime}}} u_{\alpha}^{2^{\star}} d v_{g}\right)^{2 / 2^{\star} \leq} & K_{n}^{2} \mu_{\alpha}-\alpha K_{n}^{2}\left(\int_{M} u_{\alpha}^{\theta} d v_{g}\right)^{2 / \theta} \\
& +C \int_{M \backslash B_{\delta^{\prime}}}\left|\nabla u_{\alpha}\right|^{2} d v_{g}+C \int_{M \backslash B_{\delta^{\prime}}} u_{\alpha}\left|\nabla u_{\alpha}\right| d v_{g} \\
& +C \int_{M \backslash B_{\delta^{\prime}}} u_{\alpha}^{2} d v_{g}+B\left(\int_{M} u_{\alpha}^{\theta} d v_{g}\right)^{2 / \theta}
\end{aligned}
$$

for some other constant $C>0$ independent of $\alpha$. Clearly,

$$
1-\left(\int_{B_{\delta^{\prime}}} u_{\alpha}^{2^{\star}} d v_{g}\right)^{2 / 2^{\star}} \leq \int_{M \backslash B_{\delta^{\prime}}} u_{\alpha}^{2^{\star}} d v_{g}
$$

while

$$
\int_{M \backslash B_{\delta^{\prime}}} u_{\alpha}\left|\nabla u_{\alpha}\right| d v_{g} \leq\left(\int_{M \backslash B_{\delta^{\prime}}} u_{\alpha}^{2} d v_{g}\right)^{1 / 2}\left(\int_{M \backslash B_{\delta^{\prime}}}\left|\nabla u_{\alpha}\right|^{2} d v_{g}\right)^{1 / 2}
$$

Since $\mu_{\alpha} K_{n}^{2}<1$, one gets

$$
\begin{aligned}
\alpha K_{n}^{2}-B \leq & \frac{\int_{M \backslash B_{\delta^{\prime}}} u_{\alpha}^{2^{\star}} d v_{g}}{\left(\int_{M} u_{\alpha}^{\theta} d v_{g}\right)^{2 / \theta}}+C \frac{\int_{M \backslash B_{\delta^{\prime}}}\left|\nabla u_{\alpha}\right|^{2} d v_{g}}{\left(\int_{M} u_{\alpha}^{\theta} d v_{g}\right)^{2 / \theta}}+C \frac{\int_{M \backslash B_{\delta^{\prime}}} u_{\alpha}^{2} d v_{g}}{\left(\int_{M} u_{\alpha}^{\theta} d v_{g}\right)^{2 / \theta}} \\
& +C\left(\frac{\int_{M \backslash B_{\delta^{\prime}}} u_{\alpha}^{2} d v_{g}}{\left(\int_{M} u_{\alpha}^{\theta} d v_{g}\right)^{2 / \theta}}\right)^{1 / 2}\left(\frac{\int_{M \backslash B_{\delta^{\prime}}}\left|\nabla u_{\alpha}\right|^{2} d v_{g}}{\left(\int_{M} u_{\alpha}^{\theta} d v_{g}\right)^{2 / \theta}}\right)^{1 / 2}
\end{aligned}
$$

By lemma 1,

$$
\begin{aligned}
\int_{M \backslash B_{\delta^{\prime}}} u_{\alpha}^{2} d v_{g} & \leq V_{g}\left(\sup _{M \backslash B_{\delta^{\prime}}} u_{\alpha}\right)^{2} \\
& \leq C\left(\int_{M} u_{\alpha}^{\theta} d v_{g}\right)^{2 / \theta}
\end{aligned}
$$

where $V_{g}$ stands for the volume of $M$ with respect to $g$, and $C>0$ is independent of $\alpha$. As a consequence,

$$
\frac{\int_{M \backslash B_{\delta^{\prime}}} u_{\alpha}^{2} d v_{g}}{\left(\int_{M} u_{\alpha}^{\theta} d v_{g}\right)^{2 / \theta}} \leq C
$$


Together with (1.9),

$$
\frac{\int_{M \backslash B_{\delta^{\prime}}} u_{\alpha}^{2^{\star}} d v_{g}}{\left(\int_{M} u_{\alpha}^{\theta} d v_{g}\right)^{2 / \theta}} \leq C\left(\sup _{M \backslash B_{\delta^{\prime}}} u_{\alpha}\right)^{2^{\star}-2}
$$

so that

$$
\lim _{\alpha \rightarrow+\infty} \frac{\int_{M \backslash B_{\delta^{\prime}}} u_{\alpha}^{2^{\star}} d v_{g}}{\left(\int_{M} u_{\alpha}^{\theta} d v_{g}\right)^{2 / \theta}}=0
$$

For $\delta^{\prime \prime} \in\left(0, \delta^{\prime}\right)$, let $0 \leq \eta^{\prime \prime} \leq 1$ be a smooth function on $M$ such that $\eta^{\prime \prime}=0$ on $B_{\delta^{\prime \prime}}$ and $\eta^{\prime \prime}=1$ on $M \backslash B_{\delta^{\prime \prime}}$. Mutiplying $\left(E_{\alpha}\right)$ by $\left(\eta^{\prime \prime}\right)^{2} u_{\alpha}$, and integrating over $M$, gives

$$
\int_{M}\left(\eta^{\prime \prime}\right)^{2}\left|\nabla u_{\alpha}\right|^{2} d v_{g}+2 \int_{M} \eta^{\prime \prime} u_{\alpha}\left\langle\nabla \eta^{\prime \prime}, \nabla u_{\alpha}\right\rangle d v_{g} \leq K_{n}^{-2} \int_{M}\left(\eta^{\prime \prime}\right)^{2} u_{\alpha}^{2^{\star}} d v_{g}
$$

In particular,

$$
\begin{aligned}
& \int_{M}\left(\eta^{\prime \prime}\right)^{2}\left|\nabla u_{\alpha}\right|^{2} d v_{g} \\
& \quad \leq C \int_{M}\left(\eta^{\prime \prime}\right)^{2} u_{\alpha}^{2^{\star}} d v_{g}+C\left(\int_{M}\left|\nabla \eta^{\prime \prime}\right|^{2} u_{\alpha}^{2} d v_{g}\right)^{1 / 2}\left(\int_{M}\left(\eta^{\prime \prime}\right)^{2}\left|\nabla u_{\alpha}\right|^{2} d v_{g}\right)^{1 / 2}
\end{aligned}
$$

for some constant $C>0$ independent of $\alpha$. Hence,

$$
\begin{aligned}
& \frac{\int_{M}\left(\eta^{\prime \prime}\right)^{2}\left|\nabla u_{\alpha}\right|^{2} d v_{g}}{\left(\int_{M} u_{\alpha}^{\theta} d v_{g}\right)^{2 / \theta}} \\
& \quad \leq C \frac{\int_{M}\left(\eta^{\prime \prime}\right)^{2} u_{\alpha}^{2^{\star}} d v_{g}}{\left(\int_{M} u_{\alpha}^{\theta} d v_{g}\right)^{2 / \theta}}+C\left(\frac{\int_{M}\left|\nabla \eta^{\prime \prime}\right|^{2} u_{\alpha}^{2} d v_{g}}{\left(\int_{M} u_{\alpha}^{\theta} d v_{g}\right)^{2 / \theta}}\right)^{1 / 2}\left(\frac{\int_{M}\left(\eta^{\prime \prime}\right)^{2}\left|\nabla u_{\alpha}\right|^{2} d v_{g}}{\left(\int_{M} u_{\alpha}^{\theta} d v_{g}\right)^{2 / \theta}}\right)^{1 / 2}
\end{aligned}
$$

By (1.13),

$$
\lim _{\alpha \rightarrow+\infty} \frac{\int_{M}\left(\eta^{\prime \prime}\right)^{2} u_{\alpha}^{2^{\star}} d v_{g}}{\left(\int_{M} u_{\alpha}^{\theta} d v_{g}\right)^{2 / \theta}}=0
$$

while by (1.12),

$$
\frac{\int_{M}\left|\nabla \eta^{\prime \prime}\right|^{2} u_{\alpha}^{2} d v_{g}}{\left(\int_{M} u_{\alpha}^{\theta} d v_{g}\right)^{2 / \theta}} \leq C
$$

for some $C>0$ independent of $\alpha$. Noting that

$$
\frac{\int_{M \backslash B_{\delta^{\prime}}}\left|\nabla u_{\alpha}\right|^{2} d v_{g}}{\left(\int_{M} u_{\alpha}^{\theta} d v_{g}\right)^{2 / \theta}} \leq \frac{\int_{M}\left(\eta^{\prime \prime}\right)^{2}\left|\nabla u_{\alpha}\right|^{2} d v_{g}}{\left(\int_{M} u_{\alpha}^{\theta} d v_{g}\right)^{2 / \theta}}
$$


one gets the existence of $C>0$ independent of $\alpha$ such that

$$
\frac{\int_{M \backslash B_{\delta^{\prime}}}\left|\nabla u_{\alpha}\right|^{2} d v_{g}}{\left(\int_{M} u_{\alpha}^{\theta} d v_{g}\right)^{2 / \theta}} \leq C
$$

Combining (1.11) with (1.12)-(1.14), leads to a contradiction. This ends the proof of the lemma.

We prove now Theorem 1.

Proof of Theorem 1. Given a smooth compact Riemannian $n$-manifold $(M, g)$, suppose first that its sectional curvature $K_{g}$ is nonpositive, and that the $n$-dimensional Cartan-Hadamard conjecture is true. Let $(\tilde{M}, \tilde{g})$ be the universal Riemannian covering of $(M, g)$. Then for any smooth bounded domain $\Omega$ in $\tilde{M}$,

$$
\frac{\operatorname{Area}_{\tilde{g}}(\partial \Omega)}{\operatorname{Vol}_{\tilde{g}}(\Omega)^{(n-1) / n}} \geq n\left(\frac{\omega_{n-1}}{n}\right)^{\frac{1}{n}}
$$

By standard arguments (see for instance Aubin-Druet-Hebey [2] or Hebey [17]), (1.15) implies that for any $u \in C_{c}^{\infty}(\tilde{M})$,

$$
\left(\int_{\tilde{M}}|u|^{2^{\star}} d v_{\tilde{g}}\right)^{2 / 2^{\star}} \leq K_{n}^{2} \int_{\tilde{M}}|\nabla u|^{2} d v_{\tilde{g}}
$$

Since $(M, g)$ is locally isometric to $(\tilde{M}, \tilde{g})$, for any $\theta \in[1,2],(1.16)$ implies that $\left(S_{o p t}^{\theta}\right)$ is locally valid on $(M, g)$. By lemma 2 , this implies that $\left(S_{o p t}^{\theta}\right)$ is valid on $(M, g)$. The same argument leads to the same conclusion if $K_{g}$ is a nonpositive constant, since (1.15) holds for hyperbolic space (and Euclidean space).

Conversely, let us prove that $\left(S_{o p t}^{\theta}\right)$ is false if $\theta \in[1,2), n \geq 4$ and $S_{g}>0$ somewhere on $M$. We can do this very simply. The result was also announced in Aubin and $\mathrm{Li}$ [3]. Given $x \in M$ such that $S_{g}(x)$ is positive, we let $r>0$ be such that $r<i_{g}(x)$, the injectivity radius at $x$. In geodesic normal coordinates,

$$
\frac{1}{\omega_{n-1}} \int_{S(r)} \sqrt{\operatorname{det}\left(g_{i j}\right)} d s=1-\frac{1}{6 n} S_{g}(x) r^{2}+O\left(r^{4}\right)
$$

where $S(r)$ stands for the sphere of radius $r$ and center $x$ in $M$. For $\varepsilon>0$, we define

$$
\begin{aligned}
& u_{\varepsilon}=\left(\varepsilon+r^{2}\right)^{1-n / 2}-\left(\varepsilon+\delta^{2}\right)^{1-n / 2} \text { if } r \leq \delta \\
& u_{\varepsilon}=0 \text { otherwise }
\end{aligned}
$$

where $\delta \in\left(0, i_{g}(x)\right)$ is given and $r=d_{g}(x,$.$) . Easy computations lead to$

$$
\begin{aligned}
\int_{M}\left|\nabla u_{\varepsilon}\right|^{2} d v_{g}= & \frac{(n-2)^{2} \omega_{n-1}}{2} I_{n}^{n / 2} \varepsilon^{1-n / 2} \\
& \quad \times\left(1-\frac{(n+2)}{6 n(n-4)} S_{g}(x) \varepsilon+o(\varepsilon)\right) \text { if } n>4 \\
= & \frac{(n-2)^{2} \omega_{n-1}}{2} \varepsilon^{1-n / 2} \\
& \quad \times\left(I_{n}^{n / 2}+\frac{1}{6 n} S_{g}(x) \varepsilon \log \varepsilon+o(\varepsilon \log \varepsilon)\right) \text { if } n=4
\end{aligned}
$$


and

$$
\begin{aligned}
\int_{M} u_{\varepsilon}^{2^{\star}} d v_{g} \geq & \frac{(n-2) \omega_{n-1}}{2 n} I_{n}^{n / 2} \varepsilon^{-n / 2} \\
& \times\left(1-\frac{1}{6(n-2)} S_{g}(x) \varepsilon+o(\varepsilon)\right) \quad \text { if } n>4 \\
\geq & \frac{(n-2) \omega_{n-1}}{2 n} I_{n}^{n / 2} \varepsilon^{-n / 2} \\
& \quad \times(1+o(\varepsilon \log \varepsilon)) \text { if } n=4
\end{aligned}
$$

where $I_{p}^{q}=\int_{0}^{+\infty}(1+t)^{-p} t^{q} d t$. As one can easily check,

$$
\frac{\omega_{n}}{2^{n-1} \omega_{n-1}}=I_{n}^{n / 2-1}=\frac{(n-2)}{n} I_{n}^{n / 2}
$$

Hence,

$$
\frac{(n-2)^{2} \omega_{n-1}}{2} I_{n}^{n / 2}=\frac{1}{K(n, 2)^{2}}\left(\frac{(n-2) \omega_{n-1}}{2 n} I_{n}^{n / 2}\right)^{(n-2) / n}
$$

Independently, for $\theta \in[1,2)$,

$$
\begin{aligned}
\int_{M} u_{\varepsilon}^{\theta} d v_{g} & =o\left(\varepsilon^{2-n / 2}\right) \quad \text { if } n>4 \\
& =o(\log \varepsilon) \quad \text { if } n=4
\end{aligned}
$$

Given $B \in \mathbb{R}$, this leads to

$$
\begin{aligned}
\frac{\left\|\nabla u_{\varepsilon}\right\|_{2}^{2}+B\left\|u_{\varepsilon}\right\|_{\theta}^{2}}{\left\|u_{\varepsilon}\right\|_{2^{\star}}^{2}} & \leq K_{n}^{-2}\left(1-\frac{S_{g}(x)}{n(n-4)} \varepsilon+o(\varepsilon)\right) \text { if } n>4 \\
& \leq K_{4}^{-2}\left(1+\frac{1}{8} S_{g}(x) \varepsilon \log \varepsilon+o(\varepsilon \log \varepsilon)\right) \text { if } n=4
\end{aligned}
$$

As a consequence, for $n \geq 4$ and any $B \in \mathbb{R}$,

$$
\frac{\left\|\nabla u_{\varepsilon}\right\|_{2}^{2}+B\left\|u_{\varepsilon}\right\|_{\theta}^{2}}{\left\|u_{\varepsilon}\right\|_{2^{\star}}^{2}}<\frac{1}{K_{n}^{2}}
$$

provided that $\varepsilon>0$ is small. Clearly, this ends the proof of Theorem 1 .

\section{Proof of Theorem 2}

An interesting situation where Theorem 2 is easy to get is when the manifold considered is conformally flat. This includes the case of the standard 3-sphere. The result is then an easy consequence of the following inequality obtained by Brezis and Nirenberg [6]: for $\Omega$ a smooth bounded domain in $\mathbb{R}^{3}$, and for any $u \in C_{c}^{\infty}(\Omega)$,

$$
\|u\|_{2^{\star}}^{2} \leq K_{3}^{2}\|\nabla u\|_{2}^{2}-\lambda|\Omega|^{-2 / 3}\|u\|_{2}^{2}
$$

where $|\Omega|$ stands for the volume of $\Omega$, and $\lambda>0$, known explicitly, does not depend on $\Omega$. If $\xi$ stands for the Euclidean metric, and $(M, g)$ is conformally flat, then for any $x$ in $M$, there exist $r_{x}>0$ and a smooth positive function $\varphi_{x}$ on $M$ such that in some chart at $x$ whose domain contains $\Omega_{x}=B_{x}\left(r_{x}\right)$ we have $\xi=\varphi_{x}^{-4} g$ on $\Omega_{x}$. As one can easily check, for $u \in C_{c}^{\infty}\left(\Omega_{x}\right)$,

$$
\int_{M}\left|\nabla\left(u \varphi_{x}\right)\right|^{2} d x=\int_{M}|\nabla u|^{2} d v_{g}+\frac{1}{8} \int_{M} S_{g} u^{2} d v_{g}
$$


where $S_{g}$ stands for the scalar curvature of $g$. Coming back to (2.1), for any $u \in C_{c}^{\infty}\left(\Omega_{x}\right)$

$$
\begin{aligned}
& \left(\int_{M} u^{6} d v_{g}\right)^{1 / 3}+\lambda\left|\Omega_{x}\right|^{-2 / 3} \int_{M} \frac{u^{2}}{\varphi_{x}^{4}} d v_{g} \\
& \quad \leq K_{3}^{2} \int_{M}|\nabla u|^{2} d v_{g}+\frac{1}{8} K_{3}^{2} \int_{M} S_{g} u^{2} d v_{g}
\end{aligned}
$$

Choosing $r_{x}>0$ small enough so that

$$
\lambda\left|\Omega_{x}\right|^{-2 / 3}\left(\max \varphi_{x}^{-4}\right) \geq \frac{1}{8} K_{3}^{2}\left(\max S_{g}\right)
$$

one then gets that for any $x \in M$, there exists an open neighborhood $\Omega_{x}$ of $x$ such that for any $u \in C_{c}^{\infty}\left(\Omega_{x}\right)$,

$$
\left(\int_{M} u^{6} d v_{g}\right)^{1 / 3} \leq K_{3}^{2} \int_{M}|\nabla u|^{2} d v_{g}
$$

By lemma 2, this proves the above claim.

For an arbitrary smooth compact 3 -manifold, we need to be more subtle. The argument there follows estimates that were obtained by Druet [11] in his resolution of the conjecture on sharp Sobolev constants. Clearly, it suffices to prove the result for $\theta=1$. As when proving that sharp Sobolev inequalities with lower order remainder terms are localisable (lemma 2), we proceed by contradiction. We assume therefore that for any $\alpha>0$,

$$
\inf _{H_{1}^{2}(M) \backslash\{0\}} I_{\alpha}(u)<\frac{1}{K_{3}^{2}}
$$

where

$$
I_{\alpha}(u)=\frac{\|\nabla u\|_{2}^{2}+\alpha\|u\|_{1}^{2}}{\|u\|_{6}^{2}}
$$

As in the proof of lemma 2, (2.2) leads to the existence of a minimizer $u_{\alpha} \in H_{1}^{2}(M)$ with $u_{\alpha} \geq 0$ and of norm 1 in $L^{6}(M)$. If $\mu_{\alpha}$ stands for the above infimum, one has in addition that

$$
\Delta_{g} u_{\alpha}+\alpha\left(\int_{M} u_{\alpha} d v_{g}\right) \Sigma_{\alpha}=\mu_{\alpha} u_{\alpha}^{5}
$$

where $\Sigma_{\alpha} \in L^{\infty}(M)$ is such that $0 \leq \Sigma_{\alpha} \leq 1$ and $\Sigma_{\alpha} \varphi=\varphi$ for any $\varphi \in H_{1}^{2}(M)$ having the property that $|\varphi| \leq C u_{\alpha}$ on $M$ for some constant $C>0$. Moreover, $u_{\alpha}$ is actually $C^{1, \lambda}$ for any $\lambda \in(0,1)$, and the sequence $\left(u_{\alpha}\right)$ is bounded in $H_{1}^{2}(M)$.

Multiplying $\left(E_{\alpha}\right)$ by $u_{\alpha}$, and integrating over $M$, we get that

$$
\left\|\nabla u_{\alpha}\right\|_{2}^{2}+\alpha\left\|u_{\alpha}\right\|_{1}^{2}=\mu_{\alpha}
$$

As a consequence, $\left\|u_{\alpha}\right\|_{1} \rightarrow 0$ as $\alpha \rightarrow+\infty$. By Hölder's inequality, and since $u_{\alpha}$ is of norm 1 in $L^{6}$, this implies that $\left\|u_{\alpha}\right\|_{2} \rightarrow 0$ as $\alpha \rightarrow+\infty$. By Hebey and Vaugon [19], there exists $B \in \mathbb{R}$ such that for any $u \in C^{\infty}(M)$,

$$
\|u\|_{6}^{2} \leq K_{3}^{2}\|\nabla u\|_{2}^{2}+B\|u\|_{2}^{2}
$$

Taking $u=u_{\alpha}$ in this inequality, we get

$$
1 \leq K_{3}^{2}\left\|\nabla u_{\alpha}\right\|_{2}^{2}+B\left\|u_{\alpha}\right\|_{2}^{2} \leq K_{3}^{2} \mu_{\alpha}+B\left\|u_{\alpha}\right\|_{2}^{2}
$$


Together with (2.2), this implies that

$$
\lim _{\alpha \rightarrow+\infty} \mu_{\alpha}=\frac{1}{K_{3}^{2}}
$$

Similarly,

$$
1-B\left\|u_{\alpha}\right\|_{2}^{2} \leq K_{3}^{2}\left\|\nabla u_{\alpha}\right\|_{2}^{2}=K_{3}^{2} \mu_{\alpha}-\alpha\left\|u_{\alpha}\right\|_{1}^{2}
$$

so that

$$
\lim _{\alpha \rightarrow+\infty} \alpha\left\|u_{\alpha}\right\|_{1}^{2}=0
$$

Now define a concentration point $x$ for $\left(u_{\alpha}\right)$ by the property that for any $\delta>0$,

$$
\limsup _{\alpha \rightarrow+\infty} \int_{B_{x}(\delta)} u_{\alpha}^{6} d v_{g}>0
$$

As in the proof of lemma 2, we get that, up to a subsequence, $\left(u_{\alpha}\right)$ has one and only one concentration point $x_{0}$. One may then assume that for any $\delta>0$,

$$
\lim _{\alpha \rightarrow+\infty} \int_{B_{x_{0}}(\delta)} u_{\alpha}^{6} d v_{g}=1
$$

Moreover,

$$
u_{\alpha} \rightarrow 0 \quad \text { in } C_{l o c}^{0}\left(M \backslash\left\{x_{0}\right\}\right)
$$

as $\alpha$ goes to $+\infty$.

We let $x_{\alpha} \in M$ and $\lambda_{\alpha} \in \mathbb{R}$ be such that

$$
u_{\alpha}\left(x_{\alpha}\right)=\left\|u_{\alpha}\right\|_{\infty}=\lambda_{\alpha}^{-1 / 2}
$$

According to what we just said, $x_{\alpha} \rightarrow x_{0}$ and $\lambda_{\alpha} \rightarrow 0$ as $\alpha \rightarrow+\infty$. By (2.4), noting that

$$
1=\left\|u_{\alpha}\right\|_{6}^{6} \leq\left\|u_{\alpha}\right\|_{\infty}^{5}\left\|u_{\alpha}\right\|_{1}
$$

one gets that

$$
\lim _{\alpha \rightarrow+\infty} \alpha \lambda_{\alpha}^{5 / 2}\left\|u_{\alpha}\right\|_{1}=0
$$

Here again, the proof now splits into several steps.

Step 1 . We claim that for any $R>0$,

$$
\lim _{\alpha \rightarrow+\infty} \int_{B_{x_{\alpha}}\left(R \lambda_{\alpha}\right)} u_{\alpha}^{6} d v_{g}=1-\varepsilon_{R}
$$

where $\varepsilon_{R}>0$ is such that $\varepsilon_{R} \rightarrow 0$ as $R \rightarrow+\infty$. We let $\exp _{x_{\alpha}}$ be the exponential map at $x_{\alpha}$. There clearly exists $\delta>0$, independent of $\alpha$, such that for any $\alpha, \exp _{x_{\alpha}}$ is a diffeomorphism from $B_{0}(\delta) \subset \mathbb{R}^{3}$ onto $B_{x_{\alpha}}(\delta)$. For $x \in B_{0}\left(\lambda_{\alpha}^{-1} \delta\right)$, set

$$
\begin{aligned}
& \tilde{g}_{\alpha}(x)=\left(\exp _{x_{\alpha}}^{\star} g\right)\left(\lambda_{\alpha} x\right) \\
& \tilde{u}_{\alpha}(x)=\lambda_{\alpha}^{1 / 2} u_{\alpha}\left(\exp _{x_{\alpha}}\left(\lambda_{\alpha} x\right)\right) \\
& \tilde{\Sigma}_{\alpha}=\Sigma_{\alpha}\left(\exp _{x_{\alpha}}\left(\lambda_{\alpha} x\right)\right)
\end{aligned}
$$

As we can easily check,
$\left(\tilde{E}_{\alpha}\right)$
$\Delta_{\tilde{g}_{\alpha}} \tilde{u}_{\alpha}+\alpha\left\|u_{\alpha}\right\|_{1} \lambda_{\alpha}^{5 / 2} \tilde{\Sigma}_{\alpha}=\mu_{\alpha} \tilde{u}_{\alpha}^{5}$

Moreover,

$$
\tilde{u}_{\alpha}(0)=\left\|\tilde{u}_{\alpha}\right\|_{\infty}=1
$$


and, if $\xi$ stands for the Euclidean metric of $\mathbb{R}^{3}$,

$$
\lim _{\alpha \rightarrow+\infty} \tilde{g}_{\alpha}=\xi \quad \text { in } C^{2}(K)
$$

for any compact subset $K$ of $\mathbb{R}^{3}$. By (2.7), (2.9), and theorem 8.24 of Gilbarg and Trudinger 14, $\left(\tilde{u}_{\alpha}\right)$ is equicontinuous on any compact subset of $\mathbb{R}^{3}$. By Ascoli's theorem, one gets the existence of some $\tilde{u} \in C^{0}\left(\mathbb{R}^{3}\right)$ such that for any compact subset $K$ of $\mathbb{R}^{3}$,

$$
\lim _{\alpha \rightarrow+\infty} \tilde{u}_{\alpha}=\tilde{u} \quad \text { in } L^{\infty}(K)
$$

Clearly, $\tilde{u}(0)=1$ and $\tilde{u} \not \equiv 0$. It is easy to check that $\tilde{u} \in H_{0,1}^{2}\left(\mathbb{R}^{3}\right)$, where $H_{0,1}^{2}\left(\mathbb{R}^{3}\right)$ stands for the completion of $C_{c}^{\infty}\left(\mathbb{R}^{3}\right)$ with respect to

$$
\|u\|_{H_{0,1}^{2}}=\sqrt{\int_{\mathbb{R}^{3}}|\nabla u|^{2} d x}
$$

Indeed, let $\eta \in C_{c}^{\infty}\left(\mathbb{R}^{3}\right), 0 \leq \eta \leq 1$, be such that $\eta=1$ in $B_{0}(\delta / 4)$ and $\eta=0$ in $\mathbb{R}^{3} \backslash B_{0}(\delta / 2)$. We set $\eta_{\alpha}(x)=\eta\left(\mu_{\alpha} x\right)$, and

$$
\varphi_{\alpha}(x)=\eta_{\alpha}(x) \tilde{u}_{\alpha}(x)
$$

Then, $\varphi_{\alpha} \in C_{c}^{1}\left(\mathbb{R}^{3}\right)$, and $\varphi_{\alpha} \rightarrow \tilde{u}$ in $L^{\infty}(K)$ for any compact subset $K$ of $\mathbb{R}^{3}$. Clearly, there exists $C>0$ such that for any $\alpha$,

$$
\begin{aligned}
\left\|\varphi_{\alpha}\right\|_{H_{0,1}^{2}} & \leq C \int_{\mathbb{R}^{3}}\left|\nabla \varphi_{\alpha}\right|^{2} d v_{\tilde{g}_{\alpha}} \\
& \leq C \int_{\mathbb{R}^{3}} \eta_{\alpha}^{2}\left|\nabla \tilde{u}_{\alpha}\right|^{2} d v_{\tilde{g}_{\alpha}}+C \lambda_{\alpha}^{2} \int_{\mathbb{R}^{3}}\left|\nabla \eta\left(\mu_{\alpha} x\right)\right|^{2} \tilde{u}_{\alpha}^{2} d v_{\tilde{g}_{\alpha}}
\end{aligned}
$$

On the one hand,

$$
\int_{B_{0}\left(\delta \lambda_{\alpha}^{-1}\right)} \tilde{u}_{\alpha}^{2} d v_{\tilde{g}_{\alpha}}=\lambda_{\alpha}^{-2} \int_{B_{x_{\alpha}}(\delta)} u_{\alpha}^{2} d v_{g}
$$

On the other hand,

$$
\int_{\mathbb{R}^{3}} \eta_{\alpha}^{2}\left|\tilde{u}_{\alpha}\right|^{2} d v_{\tilde{g}_{\alpha}} \leq \int_{B_{0}\left(\delta \lambda_{\alpha}^{-1}\right)}\left|\tilde{u}_{\alpha}\right|^{2} d v_{\tilde{g}_{\alpha}}=\int_{B_{x_{\alpha}}(\delta)}\left|\nabla u_{\alpha}\right|^{2} d v_{g}
$$

Hence, $\left(\varphi_{\alpha}\right)$ is bounded in $H_{0,1}^{2}\left(\mathbb{R}^{3}\right)$, and since $H_{0,1}^{2}\left(\mathbb{R}^{3}\right)$ is reflexive, $\tilde{u} \in H_{0,1}^{2}\left(\mathbb{R}^{3}\right)$. This proves the above assertion. By passing to the limit as $\alpha$ goes to $+\infty$ in $\left(\tilde{E}_{\alpha}\right)$, according to $(2.3),(2.7),(2.10)$, and $(2.11)$, one now gets that $\tilde{u}$ is a solution of

$$
\Delta_{\xi} \tilde{u}=\frac{1}{K_{3}^{2}} \tilde{u}^{5}
$$

By Caffarelli-Gidas-Spruck [7], or also Obata [23],

$$
\tilde{u}(x)=\left(\frac{3 K_{3}^{2}}{3 K_{3}^{2}+|x|^{2}}\right)^{1 / 2}
$$

since $\tilde{u}(0)=1$. Noting that $\tilde{u}$ is of norm 1 in $L^{6}\left(\mathbb{R}^{3}\right)$, and that for any $R>0$,

$$
\int_{B_{x_{\alpha}}\left(R \lambda_{\alpha}\right)} u_{\alpha}^{6} d v_{g}=\int_{B_{0}(R)} \tilde{u}_{\alpha}^{6} d v_{\tilde{g}_{\alpha}}
$$

one gets that

$$
\lim _{\alpha \rightarrow+\infty} \int_{B_{x_{\alpha}}\left(R \lambda_{\alpha}\right)} u_{\alpha}^{6} d v_{g}=1-\int_{\mathbb{R}^{3} \backslash B_{0}(R)} \tilde{u}^{6} d x
$$


Clearly, this proves (2.8) and the claim we made in step 1.

Step 2. We claim that there exists $C>0$, independent of $\alpha$, such that for any $\alpha$, and any $x$,

$$
d_{g}\left(x_{\alpha}, x\right)^{1 / 2} u_{\alpha}(x) \leq C
$$

where $d_{g}$ stands for the distance with respect to $g$. In order to prove this, set

$$
v_{\alpha}(x)=d_{g}\left(x_{\alpha}, x\right)^{1 / 2} u_{\alpha}(x)
$$

and assume by contradiction that, for some subsequence,

$$
\lim _{\alpha \rightarrow+\infty}\left\|v_{\alpha}\right\|_{\infty}=+\infty
$$

Let $y_{\alpha}$ be some point in $M$ where $v_{\alpha}$ is maximum. By (2.6), $y_{\alpha} \rightarrow x_{0}$ as $\alpha \rightarrow+\infty$, while by $(2.14)$,

$$
\lim _{\alpha \rightarrow+\infty} \frac{d_{g}\left(x_{\alpha}, y_{\alpha}\right)}{\lambda_{\alpha}}=+\infty
$$

Fix $\delta>0$ small, and set

$$
\Omega_{\alpha}=u_{\alpha}\left(y_{\alpha}\right)^{2} \exp _{y_{\alpha}}^{-1}\left(B_{x_{\alpha}}(\delta)\right)
$$

For $x \in \Omega_{\alpha}$, define

$$
\tilde{v}_{\alpha}(x)=u_{\alpha}\left(y_{\alpha}\right)^{-1} u_{\alpha}\left(\exp _{y_{\alpha}}\left(u_{\alpha}\left(y_{\alpha}\right)^{-2} x\right)\right)
$$

and

$$
h_{\alpha}(x)=\left(\exp _{y_{\alpha}}^{\star} g\right)\left(u_{\alpha}\left(y_{\alpha}\right)^{-2} x\right)
$$

Clearly,

$$
\lim _{\alpha \rightarrow+\infty} h_{\alpha}=\xi \text { in } C^{2}\left(B_{0}(2)\right)
$$

Moreover, as one can easily check,

$$
\Delta_{h_{\alpha}} \tilde{v}_{\alpha} \leq \mu_{\alpha} \tilde{v}_{\alpha}^{5}
$$

Since $v_{\alpha}\left(y_{\alpha}\right)$ goes to $+\infty$ as $\alpha$ goes to $+\infty$, using (2.14), one gets that for $\alpha$ large, and all $x \in B_{0}(2)$,

$$
d_{g}\left(x_{\alpha}, \exp _{y_{\alpha}}\left(u_{\alpha}\left(y_{\alpha}\right)^{-2} x\right)\right) \geq \frac{1}{2} d_{g}\left(x_{\alpha}, y_{\alpha}\right)
$$

This implies that

$$
\begin{aligned}
\tilde{v}_{\alpha}(x) & \leq \sqrt{2} d_{g}\left(x_{\alpha}, y_{\alpha}\right)^{-1 / 2} u_{\alpha}\left(y_{\alpha}\right)^{-1} v_{\alpha}\left(\exp _{y_{\alpha}}\left(u_{\alpha}\left(y_{\alpha}\right)^{-2} x\right)\right) \\
& \leq \sqrt{2} d_{g}\left(x_{\alpha}, y_{\alpha}\right)^{-1 / 2} u_{\alpha}\left(y_{\alpha}\right)^{-1} v_{\alpha}\left(y_{\alpha}\right)
\end{aligned}
$$

so that for $\alpha$ large,

$$
\sup _{x \in B_{0}(2)} \tilde{v}_{\alpha}(x) \leq \sqrt{2}
$$

By (2.15) and (2.18), given $R>0$, and for $\alpha$ large,

$$
B_{y_{\alpha}}\left(2 u_{\alpha}\left(y_{\alpha}\right)^{-2}\right) \cap B_{x_{\alpha}}\left(R \lambda_{\alpha}\right)=\emptyset
$$

Noting that

$$
\int_{B_{0}(2)} \tilde{v}_{\alpha}^{6} d v_{h_{\alpha}}=\int_{B_{y_{\alpha}}\left(2 u_{\alpha}\left(y_{\alpha}\right)^{-2}\right)} u_{\alpha}^{6} d v_{g}
$$


and using (2.8), one gets that

$$
\lim _{\alpha \rightarrow+\infty} \int_{B_{0}(2)} \tilde{v}_{\alpha}^{6} d v_{h_{\alpha}}=0
$$

By (2.16), (2.17), (2.19), (2.20), and the De Giorgi-Nash-Moser theory, as used in lemma 1, we get

$$
\lim _{\alpha \rightarrow+\infty} \sup _{x \in B_{0}(1)} \tilde{v}_{\alpha}(x)=0
$$

But $\tilde{v}_{\alpha}(0)=1$, so that (2.14) must be false. This proves (2.13) and the claim we made in step 2 .

Step 3. We prove the theorem, showing that (2.2) leads to a contradiction. We take $\delta>0$ small (to be fixed later on), and for any $\alpha$, we let $\eta_{\alpha} \in C_{c}^{\infty}\left(B_{x_{\alpha}}(4 \delta)\right)$ be such that $0 \leq \eta_{\alpha} \leq 1, \eta_{\alpha}=1$ in $B_{x_{\alpha}}(2 \delta)$, and $\left|\nabla \eta_{\alpha}\right| \leq C / \delta$. Here, and in what follows, $C$ denotes a constant independent of $\alpha$ and $\delta$. By Brezis and Nirenberg [6], inequality (2.1), and passing through geodesic normal coordinates,

$$
\begin{gathered}
\left(\int_{B_{x_{\alpha}}(2 \delta)} u_{\alpha}^{6} d x\right)^{1 / 3} \leq K_{3}^{2} \int_{B_{x_{\alpha}}(4 \delta)}\left|\nabla\left(\eta_{\alpha} u_{\alpha}\right)\right|_{\xi}^{2} d x \\
-\frac{\lambda}{\delta^{2}} \int_{B_{x_{\alpha}}(4 \delta)}\left(\eta_{\alpha} u_{\alpha}\right)^{2} d x
\end{gathered}
$$

where $\lambda>0$ does not depend on $\alpha$ and $\delta$. When confusion is possible, we write $|\cdot|_{\xi}$ and $|\cdot|_{g}$ to specify the metric with respect to which norms are taken. Starting from the Cartan expansion of $g$ in such coordinates, we get

$$
\left|\nabla\left(\eta_{\alpha} u_{\alpha}\right)\right|_{\xi}^{2} \leq\left(1+C d_{g}\left(x_{\alpha}, x\right)^{2}\right)\left|\nabla\left(\eta_{\alpha} u_{\alpha}\right)\right|_{g}^{2}
$$

and

$$
\left(1-C d_{g}\left(x_{\alpha}, x\right)^{2}\right) d v_{g} \leq d x \leq\left(1+C d_{g}\left(x_{\alpha}, x\right)^{2}\right) d v_{g}
$$

Hence,

$$
\int_{B_{x_{\alpha}}(4 \delta)}\left|\nabla\left(\eta_{\alpha} u_{\alpha}\right)\right|_{\xi}^{2} d x \leq \int_{B_{x_{\alpha}}(4 \delta)}\left(1+C d_{g}\left(x_{\alpha}, x\right)^{2}\right)\left|\nabla\left(\eta_{\alpha} u_{\alpha}\right)\right|_{g}^{2} d v_{g}
$$

On the one hand,

$$
\begin{gathered}
\int_{B_{x_{\alpha}}(4 \delta)}\left|\nabla\left(\eta_{\alpha} u_{\alpha}\right)\right|_{g}^{2} d v_{g} \leq \int_{M}\left|\nabla u_{\alpha}\right|_{g}^{2} d v_{g}+\frac{C}{\delta^{2}} \int_{M \backslash B_{x_{\alpha}}(2 \delta)} u_{\alpha}^{2} d v_{g} \\
+\frac{C}{\delta} \int_{M \backslash B_{x_{\alpha}}(2 \delta)} u_{\alpha}\left|\nabla u_{\alpha}\right| d v_{g}
\end{gathered}
$$

Multiplying $\left(E_{\alpha}\right)$ by $u_{\alpha}$, and integrating over $M$, gives

$$
\begin{aligned}
& \int_{B_{x_{\alpha}}(4 \delta)}\left|\nabla\left(\eta_{\alpha} u_{\alpha}\right)\right|_{g}^{2} d v_{g} \leq \mu_{\alpha}-\alpha\left(\int_{M} u_{\alpha} d v_{g}\right)^{2} \\
& \quad+\frac{C}{\delta^{2}} \int_{M \backslash B_{x_{\alpha}}(2 \delta)} u_{\alpha}^{2} d v_{g}+\frac{C}{\delta} \int_{M \backslash B_{x_{\alpha}}(2 \delta)} u_{\alpha}\left|\nabla u_{\alpha}\right|_{g} d v_{g}
\end{aligned}
$$


On the other hand,

$$
\begin{gathered}
\int_{B_{x_{\alpha}}(4 \delta)} d_{g}\left(x_{\alpha}, x\right)^{2}\left|\nabla\left(\eta_{\alpha} u_{\alpha}\right)\right|_{g}^{2} d v_{g} \leq C \int_{M \backslash B_{x_{\alpha}}(2 \delta)} u_{\alpha}^{2} d v_{g} \\
+2 \int_{B_{x_{\alpha}}(4 \delta)} \eta_{\alpha}^{2}\left|\nabla u_{\alpha}\right|_{g}^{2} d_{g}\left(x_{\alpha}, x\right)^{2} d v_{g}
\end{gathered}
$$

Integrating by parts, and using $\left(E_{\alpha}\right)$, we get

$$
\begin{aligned}
\int_{B_{x_{\alpha}}(4 \delta)} & \eta_{\alpha}^{2}\left|\nabla u_{\alpha}\right|_{g}^{2} d_{g}\left(x_{\alpha}, x\right)^{2} d v_{g} \\
\leq & C \int_{B_{x_{\alpha}}(4 \delta)} d_{g}\left(x_{\alpha}, x\right)^{2} \eta_{\alpha}^{2} u_{\alpha}^{6} d v_{g}+C \int_{M \backslash B_{x_{\alpha}}(2 \delta)} u_{\alpha}\left|\nabla u_{\alpha}\right|_{g} d v_{g} \\
& +C \int_{M \backslash B_{x_{\alpha}}(2 \delta)} u_{\alpha}^{2} d v_{g}+C \int_{B_{x_{\alpha}}(4 \delta)} \eta_{\alpha}^{2} u_{\alpha}^{2} d v_{g}
\end{aligned}
$$

By (2.13),

$$
\int_{B_{x_{\alpha}}(4 \delta)} d_{g}\left(x_{\alpha}, x\right)^{2} \eta_{\alpha}^{2} u_{\alpha}^{6} d v_{g} \leq C \int_{B_{x_{\alpha}}(4 \delta)} \eta_{\alpha}^{2} u_{\alpha}^{2} d v_{g}
$$

Combining (2.24), (2.25), and (2.26), we get

$$
\begin{gathered}
\int_{B_{x_{\alpha}}(4 \delta)} d_{g}\left(x_{\alpha}, x\right)^{2}\left|\nabla\left(\eta_{\alpha} u_{\alpha}\right)\right|_{g}^{2} d v_{g} \leq C \int_{B_{x_{\alpha}}(4 \delta)} \eta_{\alpha}^{2} u_{\alpha}^{2} d v_{g} \\
+\frac{C}{\delta} \int_{M \backslash B_{x_{\alpha}}(2 \delta)} u_{\alpha}\left|\nabla u_{\alpha}\right|_{g} d v_{g}+\frac{C}{\delta^{2}} \int_{M \backslash B_{x_{\alpha}}(2 \delta)} u_{\alpha}^{2} d v_{g}
\end{gathered}
$$

Independently,

$$
\int_{B_{x_{\alpha}}(2 \delta)} u_{\alpha}^{6} d x \geq \int_{B_{x_{\alpha}}(2 \delta)} u_{\alpha}^{6} d v_{g}-C \int_{B_{x_{\alpha}}(2 \delta)} d_{g}\left(x_{\alpha}, x\right)^{2} u_{\alpha}^{6} d v_{g}
$$

so that, again by (2.13),

$$
\int_{B_{x_{\alpha}}(2 \delta)} u_{\alpha}^{6} d x \geq \int_{B_{x_{\alpha}}(2 \delta)} u_{\alpha}^{6} d v_{g}-C \int_{B_{x_{\alpha}}(2 \delta)} u_{\alpha}^{2} d v_{g}
$$

For $\alpha$ large, noting that $B_{x_{0}}(\delta) \subset B_{x_{\alpha}}(2 \delta)$, one gets from (2.5) and the fact that $\left\|u_{\alpha}\right\|_{2} \rightarrow 0$ as $\alpha \rightarrow+\infty$, that the right hand side in this inequality is positive. Since it is also less than 1 ,

$$
\left(\int_{B_{x_{\alpha}}(2 \delta)} u_{\alpha}^{6} d x\right)^{1 / 3} \geq \int_{B_{x_{\alpha}}(2 \delta)} u_{\alpha}^{6} d v_{g}-C \int_{B_{x_{\alpha}}(2 \delta)} u_{\alpha}^{2} d v_{g}
$$

and

$$
\left(\int_{B_{x_{\alpha}}(2 \delta)} u_{\alpha}^{6} d x\right)^{1 / 3} \geq 1-\int_{M \backslash B_{x_{\alpha}}(2 \delta)} u_{\alpha}^{6} d v_{g}-C \int_{B_{x_{\alpha}}(4 \delta)} \eta_{\alpha}^{2} u_{\alpha}^{2} d v_{g}
$$


By $(2.2),(2.21),(2.22),(2.23),(2.27)$, and (2.28), one gets

$$
\begin{aligned}
\alpha K_{3}^{2}\left(\int_{M} u_{\alpha} d v_{g}\right)^{2} & \\
\leq & \int_{M \backslash B_{x_{\alpha}}(2 \delta)} u_{\alpha}^{6} d v_{g}+\frac{C}{\delta^{2}} \int_{M \backslash B_{x_{\alpha}}(2 \delta)} u_{\alpha}^{2} d v_{g} \\
& +\frac{C}{\delta} \int_{M \backslash B_{x_{\alpha}}(2 \delta)} u_{\alpha}\left|\nabla u_{\alpha}\right| d v_{g}+\left(C-\frac{\lambda}{\delta^{2}}\right) \int_{B_{x_{\alpha}}(4 \delta)} \eta_{\alpha}^{2} u_{\alpha}^{2} d v_{g}
\end{aligned}
$$

We now fix $\delta>0$ sufficiently small so that

$$
C-\frac{\lambda}{\delta^{2}}<0
$$

Noting that $B_{x_{0}}(\delta) \subset B_{x_{\alpha}}(2 \delta)$, and writing by Hölder's inequality that

$$
\int_{M \backslash B_{x_{\alpha}}(2 \delta)} u_{\alpha}\left|\nabla u_{\alpha}\right| d v_{g} \leq \sqrt{\int_{M \backslash B_{x_{\alpha}}(2 \delta)} u_{\alpha}^{2} d v_{g}} \sqrt{\int_{M \backslash B_{x_{\alpha}}(2 \delta)}\left|\nabla u_{\alpha}\right|^{2} d v_{g}}
$$

one gets with (2.29) the existence of some constant $C>0$, independent of $\alpha$, such that

$$
\begin{aligned}
\alpha K_{3}^{2} & \leq \frac{\int_{M \backslash B_{x_{0}}(\delta)} u_{\alpha}^{6} d v_{g}}{\left(\int_{M} u_{\alpha} d v_{g}\right)^{2}}+C \frac{\int_{M \backslash B_{x_{0}}(\delta)} u_{\alpha}^{2} d v_{g}}{\left(\int_{M} u_{\alpha} d v_{g}\right)^{2}} \\
& +C\left(\frac{\int_{M \backslash B_{x_{0}}(\delta)} u_{\alpha}^{2} d v_{g}}{\left(\int_{M} u_{\alpha} d v_{g}\right)^{2}}\right)^{1 / 2}\left(\frac{\int_{M \backslash B_{x_{0}}(\delta)}\left|\nabla u_{\alpha}\right|^{2} d v_{g}}{\left(\int_{M} u_{\alpha} d v_{g}\right)^{2}}\right)^{1 / 2}
\end{aligned}
$$

As in the proof of lemma 2, see (1.12)-(1.14), the right hand side in (2.30) is bounded by some positive constant independent of $\alpha$. Since the left hand side of (2.30) goes to $+\infty$ as $\alpha$ goes to $+\infty$, we get a contradiction. This ends the proof of Theorem 2 .

\section{Proof of Theorem 3}

Given $\alpha>0$, let $I_{\alpha}$ be the functional defined on $H_{1}^{2}(M)$ by

$$
I_{\alpha}(u)=\|\nabla u\|_{2}^{2}+\alpha\|u\|_{2}^{2}
$$

and let $\mathcal{H}$ be the set of functions $u \in H_{1}^{2}(M)$ such that $\|u\|_{2^{\star}}=1$. According to the definition of $B_{0}(g)$, if $\alpha<B_{0}(g) K_{n}^{-2}$, then

$$
\inf _{u \in \mathcal{H}} I_{\alpha}(u)<\frac{1}{K_{n}^{2}}
$$

By standard arguments, this leads to the existence of a smooth and positive solution $u_{\alpha} \in \mathcal{H}$ of the equation

$$
\Delta_{g} u_{\alpha}+\alpha u_{\alpha}=\lambda_{\alpha} u_{\alpha}^{2^{\star}-1}
$$

where $\lambda_{\alpha}$ is the infimum in the left hand side of (3.1). Suppose now that $\left(S_{\text {opt }}^{1}\right)$ is true, and let $B$ be some constant in $\left(S_{o p t}^{1}\right)$. Multiplying (3.2) by $u_{\alpha}$, and integrating over $M$, gives us

$$
\begin{aligned}
1 & =\left\|u_{\alpha}\right\|_{2^{\star}}^{2} \\
& \leq K_{n}^{2}\left\|\nabla u_{\alpha}\right\|_{2}^{2}+B\left\|u_{\alpha}\right\|_{1}^{2} \\
& =\lambda_{\alpha} K_{n}^{2}-\alpha K_{n}^{2}\left\|u_{\alpha}\right\|_{2}^{2}+B\left\|u_{\alpha}\right\|_{1}^{2}
\end{aligned}
$$


Since $\lambda_{\alpha} K_{n}^{2}<1$, and $\alpha K_{n}^{2}<B_{0}(g)$, it follows that

$$
\left\|u_{\alpha}\right\|_{2}^{2} \leq \frac{B}{\alpha K_{n}^{2}}\left\|u_{\alpha}\right\|_{1}^{2} \leq \frac{2 B}{B_{0}(g)}\left\|u_{\alpha}\right\|_{1}^{2}
$$

for $\alpha$ close to $B_{0}(g) K_{n}^{-2}$. Independently, integrating (3.2) gives that

$$
\alpha\left\|u_{\alpha}\right\|_{1}=\lambda_{\alpha}\left\|u_{\alpha}\right\|_{2^{\star}-1}^{2^{\star}-1}
$$

When $n \geq 6$ we have $2^{\star}-1 \leq 2$, and by Hölder's inequality,

$$
\left\|u_{\alpha}\right\|_{2^{\star}-1}^{2^{\star}-1} \leq\left\|u_{\alpha}\right\|_{2}^{\frac{n+2}{n-2}} V_{g}^{\frac{n-6}{2(n-2)}}
$$

Combining (3.3) and (3.4), we get

$$
\left\|u_{\alpha}\right\|_{2}^{2} \leq \frac{2 B \lambda_{\alpha}^{2}}{\alpha^{2} B_{0}(g)} V_{g}^{\frac{n-6}{(n-2)}}\left\|u_{\alpha}\right\|_{2}^{\frac{2(n+2)}{n-2}}
$$

Clearly, this leads to the existence of $C>0$, independent of $\alpha$, such that

$$
\left\|u_{\alpha}\right\|_{2} \geq C
$$

If $n=5$, we write, by Hölder's inequality,

$$
\left\|u_{\alpha}\right\|_{2^{\star}-1}^{2^{\star}-1} \leq\left\|u_{\alpha}\right\|_{2}^{\frac{3}{2}}\left\|u_{\alpha}\right\|_{2^{\star}}^{\frac{5}{6}}
$$

Since $u_{\alpha} \in \mathcal{H}$, it follows that $\left\|u_{\alpha}\right\|_{2^{\star}}=1$, and one gets

$$
\left\|u_{\alpha}\right\|_{2^{\star}-1}^{2^{\star}-1} \leq\left\|u_{\alpha}\right\|_{2}^{\frac{3}{2}}
$$

Combining (3.3) and (3.6), we find that

$$
\left\|u_{\alpha}\right\|_{2}^{2} \leq \frac{2 B \lambda_{\alpha}^{2}}{\alpha^{2} B_{0}(g)}\left\|u_{\alpha}\right\|_{2}^{3}
$$

Hence, again, there exists $C>0$, independent of $\alpha$, such that

$$
\left\|u_{\alpha}\right\|_{2} \geq C
$$

We now let $\alpha$ go to $B_{0}(g) K_{n}^{-2}$. Clearly, $\left(u_{\alpha}\right)$ is a bounded sequence in $H_{1}^{2}(M)$. Up to a subsequence, it then converges weakly to some $u$ in $H_{1}^{2}(M)$, and strongly to $u$ in $L^{p}(M), p<2^{\star}$. Moreover, one may also assume that $\lambda_{\alpha} \rightarrow \lambda$. By (3.5) and (3.7), $u$ is nonzero. Standard arguments then give that $u$ is a smooth positive solution of the equation

$$
K_{n}^{2} \Delta_{g} u+B_{0}(g) u=\lambda K_{n}^{2} u^{2^{\star}-1}
$$

Clearly, $\|u\|_{2^{\star}} \leq 1$. Set

$$
\alpha_{0}=\frac{B_{0}(g)}{K_{n}^{2}} \quad \text { and } \quad v=\frac{u}{\|u\|_{2^{\star}}}
$$

Then, $I_{\alpha_{0}}(v) \geq K_{n}^{-2}$, and hence, by (3.8),

$$
1 \leq \lambda K_{n}^{2}\|u\|_{2^{\star}}^{2^{\star}-2}
$$

Since $\lambda K_{n}^{2} \leq 1$ and $\|u\|_{2^{\star}} \leq 1$, this implies that $\lambda K_{n}^{2}=1$ and $\|u\|_{2^{\star}}=1$. In particular, $u$ is a nonzero extremal function of $\left(S_{O P T}^{2}\right)$. The theorem is proved. 


\section{REFERENCES}

[1] Aubin, T., Problèmes isopérimétriques et espaces de Sobolev, Journal of Differential Geometry, 11, 1976, 573-598. MR 56:6711

[2] Aubin, T., Druet, O. and Hebey, E., Best constants in Sobolev inequalities for compact manifolds of nonpositive curvature, Comptes Rendus de l'Académie des Sciences, Paris, Sér. I Math. 326, 1998, 1117-1121. MR 99j:53042

[3] Aubin, T. and Li, Y.Y., Sur la meilleure constante dans l'inégalité de Sobolev, Comptes Rendus de l'Académie des Sciences, Paris, Sér. I Math. 328, 1999, 135-138. MR 2000a:46048

[4] Beckner, W., Sharp Sobolev inequalities on the sphere and the Moser-Trudinger inequality, Annals of Mathematics, 138, 1993, 213-242. MR 94m:58232

[5] Brezis, H. and Lieb, E.H., Sobolev inequalities with remainder terms, Journal of Functional Analysis, 62, 1985, 73-86. MR 86i:46033

[6] Brezis, H. and Nirenberg, L., Positive solutions of nonlinear elliptic equations involving critical Sobolev exponents, Communications on Pure and Applied Mathematics, 36, 1983, 437-477. MR 84h:35059

[7] Caffarelli, L.A., Gidas, B. and Spruck, J., Asymptotic symmetry and local behavior of semilinear elliptic equations with critical Sobolev growth, Communications on Pure and Applied Mathematics, 42, 1989, 271-297. MR 90c:35075.

[8] Croke, C.B., A sharp four dimensional isoperimetric inequality, Comment. Math. Helvetici, 59, 1984, 187-192. MR 85f:53060

[9] Djadli, Z. and Druet, O., Extremal functions and Sobolev inequalities on compact manifolds, Preprint of the university of Cergy-Pontoise, Volume 13, April 1999.

[10] Druet, O., Optimal Sobolev inequalities of arbitrary order on compact Riemannian manifolds, Journal of Functional Analysis, 159, 1998, 217-242. MR 99m:53076

[11] Druet, O., The best constants problem in Sobolev inequalities, Mathematische Annalen, 314, 1999, 327-346. MR 2000d:58033

[12] Druet, O., Hebey, E. and Vaugon, M., Optimal Nash's inequalities on Riemannian manifolds: the influence of geometry, International Mathematics Research Notices, 1995, 735-779. CMP 99:16

[13] Druet, O., Hebey, E. and Vaugon, M., Sharp Sobolev inequalities with lower order remainder terms, Preprint of the university of Cergy-Pontoise, Volume 3, January 1999.

[14] Gilbarg, D. and Trudinger, N.S., Elliptic partial differential equations of second order, Grundlehren der Mathematischen Wissenschaften, 224, Springer-Verlag, Second Edition, 1983. MR 86c:35035

[15] Hebey, E., Sobolev spaces on Riemannian manifolds, Lecture Notes in Mathematics, 1635, Springer-Verlag, 1996. MR 98k:46049

[16] Hebey, E., Fonctions extrémales pour une inégalité de Sobolev optimale dans la classe conforme de la sphère, Journal de Mathématiques Pures et Appliquées, 77, 1998, 721-733. MR 98j:58223

[17] Hebey, E., Nonlinear analysis on manifolds: Sobolev spaces and inequalities, CIMS Lecture Notes, Courant Institute of Mathematical Sciences, Volume 5, 1999. MR 2000e:58011

[18] Hebey, E., Humbert, E. and Vaugon, M., On the existence of extremal functions for the optimal $H_{1}^{2}$-Sobolev inequality after Djadli and Druet, Preprint, 1999.

[19] Hebey, E. and Vaugon, M., Meilleures constantes dans le théorème d'inclusion de Sobolev, Annales de l'Institut Henri Poincaré, Analyse Non Linéaire, 13, 1996, 57-93. MR 96m:46054

[20] Hebey, E. and Vaugon, M., The best constant problem in the Sobolev embedding theorem for complete Riemannian manifolds, Duke Mathematical Journal, 79, 1995, 235-279. MR 96c:53057

[21] Humbert, E., Best constants in the $L^{2}$-Nash inequality, Preprint, 1999.

[22] Kleiner, B., An isoperimetric comparison theorem, Inventiones Mathematicae, 108, 1992, 37-47. MR 92m:53056

[23] Obata, M., The conjectures on conformal transformations of Riemannian manifolds, Journal of Differential Geometry, 6, 1971, 247-258. MR 46:2601

[24] Talenti, G., Best constants in Sobolev inequality, Ann. di Matem. Pura ed Appl., 110, 1976, 353-372. MR 57:3846 
[25] Trudinger, N.S., Remarks concerning the conformal deformation of Riemannian structures on compact manifolds, Ann. Scuola Norm. Sup. Pisa, 22, 1968, 265-274. MR 39:2093

[26] Weil, A., Sur les surfaces à courbure négative, Comptes Rendus de l'Académie des Sciences de Paris, 182, 1926, 1069-1071.

Université de Cergy-Pontoise, Département de Mathématiques, Site de Saint-Martin, 2 avenue Adolphe Chauvin, 95302 Cergy-Pontoise cedex, France

E-mail address: Olivier.Druet@math.u-cergy.fr

Université de Cergy-Pontoise, Département de Mathématiques, Site de Saint-Martin, 2 avenue Adolphe Chauvin, 95302 Cergy-Pontoise cedex, France

E-mail address: Emmanuel.Hebey@math.u-cergy.fr

Université Pierre et Marie Curie, Département de Mathématiques, 4 place Jussieu, 75252 Paris CedeX 05, France

E-mail address: vaugon@math.jussieu.fr 